\title{
Time-varying entry heating profile replication with a rotating arc jet test article
}

\author{
Jay H. Grinstead and Ethiraj Venkatapathy \\ NASA Ames Research Center \\ Moffett Field, CA 94035
}

Eric A. Noyes, Jeffrey J. Mach, and Daniel M. Empey

Jacobs Technology, Inc

NASA Ames Research Center

Moffett Field, CA 94035

Todd R. White

ERC, Inc

NASA Ames Research Center

Moffett Field, CA 94035

Arc jets are the only means to validate the most critical performance parameters of a spacecraft heat shield's thermal protection system (TPS) at conditions simulating atmospheric entry. Arc jet testing of thermal protection materials is conducted by exposing a small sample to the arc jet stream for a prescribed duration. Facility operating conditions, test model size and shape, and distance from nozzle exit are specified to apply a prescribed constant heat flux and surface pressure to an instrumented test model. The data from several tests at a variety of (constant) flow conditions are used to develop and validate material response simulations employed for the design of a vehicle's thermal protection system. In flight, however, a spacecraft's heat shield encounters time-varying conditions where the heat flux increases, peaks, and then recedes as the vehicle descends through the atmosphere. A material's response to time-varying conditions may be different than the response to the constant condition of an arc jet test, however. For example, coking observed in Apollo-era flight tests of Avcoat has not been seen in prior or subsequent arc jet tests. Simulations developed from constant-condition arc jet testing may not capture critical thermophysical phenomena that, if known, could ultimately influence design decisions. Attempts to replicate the time-dependent aerothermal environment of an entry event by concurrently varying the flow rate(s) and arc current during a test have proven to be difficult, expensive, and only partially successful.

An alternative approach is to operate the facility at a constant condition but take advantage of the varying heat flux and pressure distribution over the surface of a curved test model. The concept utilizes a cylindrical arc jet test model that rotates on its axis, perpendicular to the flow direction, during a test run (Fig. 1a). The heat flux and pressure at a single point on the model will increase to their peak values then decrease as a function of time as the point rotates towards and away from the stagnation point. The model size and (constant) arc jet operating condition are chosen such that the stagnation point heat flux matches the anticipated peak heat flux at a targeted location on an entry vehicle's heat shield. Since the convective heat flux in flight spans from zero to its maximum and back to nearly zero (Fig. 1b), the angular direction and instantaneous rate at which the model is rotated will be programmed to realize a time-accurate heat flux 


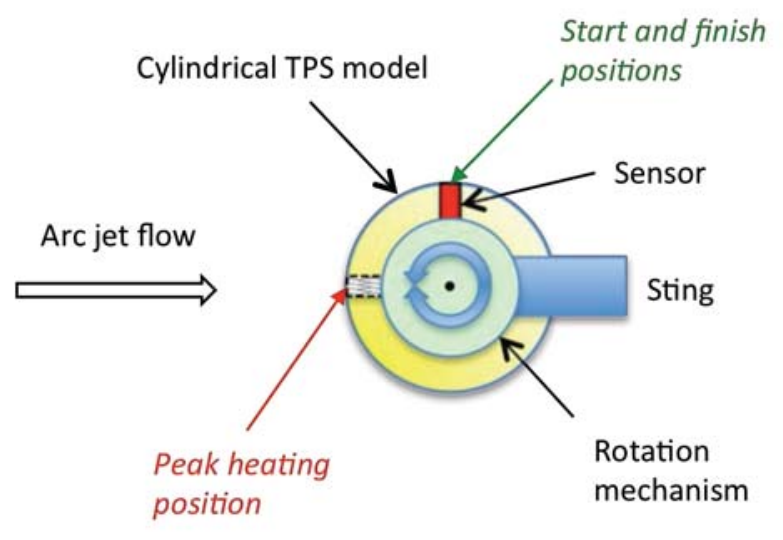

a)

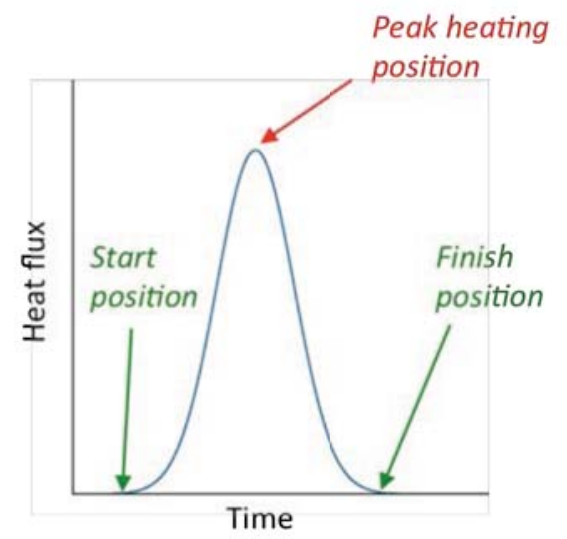

b)

Figure 1: a) Rotating arc jet test model concept. b) Time-dependent heat flux profile at sensor location on rotating test model.

profile that maps, as closely as possible, to the profile of the targeted location on the vehicle.

The MSL Entry, Descent, and Landing Instrumentation (MEDLI) project for NASA's Mars Science Laboratory (MSL) mission [1] inspired development of this concept. The forebody heat shield of the MSL entry vehicle was embedded with MISPs (MEDLI Integrated Sensor Plugs) at several locations to measure surface pressure, in-depth temperatures, and recession of the PICA (Phenolic Impregnated Carbon Ablator) heat shield material during hypersonic entry. The MISP technology was a natural fit for demonstration of the rotating test model approach as authentic time-varying conditions can applied to the integrated sensor plug to approximate the MSL flight environment.

The arc jet test article consists of 4.5" dia. cylindrical PICA model attached to an electromechanical mechanism that rotates the model. The PICA model was fitted with one MISP midway along the cylinder axis. The test model rotation mechanism is contained within a water-cooled housing attached to a facility sting arm. A controller, located remotely from the mechanism, drives a stepper motor inside the housing. A shaft encoder provides angular feedback to the controller. Figure 2 shows the test article mechanism, TPS model, and installation in an arc jet facility. The test article assembly is positioned in the facility such that the MISP lies at the stagnation point of the flow when the mechanism is rotated to achieve peak heat flux.

With constant-condition arc jet testing, cold wall heat flux and pressure measurements typically are used to anchor engineering or high-fidelity simulations that establish boundary conditions of the applied environment. A similar approach is used for the rotating test article, though the boundary conditions are time dependent. A nonrotating, instrumented cylindrical copper model of the same dimensions as the PICA models was also built and used to characterize the heat flux and pressure distribution around the cylinder. The copper cylinder was fitted with three water-cooled SchmidtBoelter heat flux gauges and pressure ports and attached to the test article mechanism. The gauge and port locations were clocked at $0^{\circ}, 45^{\circ}$, and $90^{\circ}$ to the stagnation point. The heat flux and pressure measurements are used to validate computational simulations of the test configuration (model geometry, flow conditions). The simulation results provide the location-dependent heat flux and pressure boundary conditions over the cylinder that 


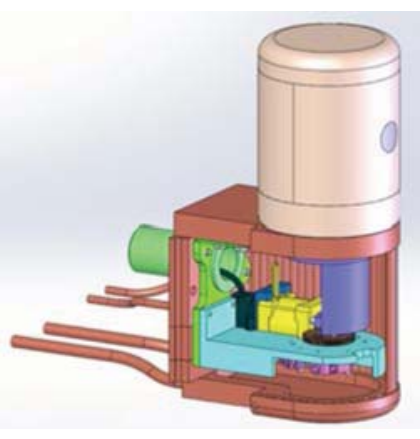

a)

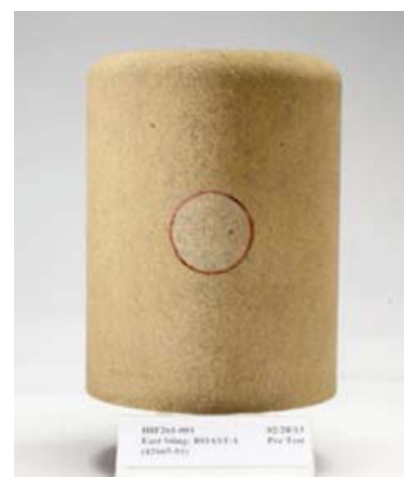

b)

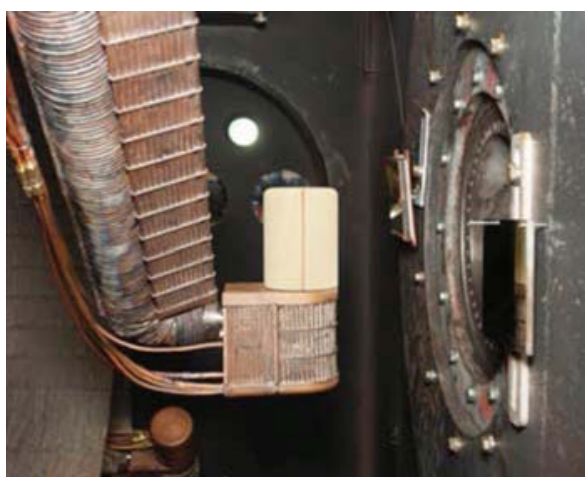

c)

Figure 2: a) Rotating arc jet test model mechanism. b) Cylindrical PICA test article with embedded MISP. c) Model and test article installed in arc jet facility.

\begin{tabular}{|l|l|}
\hline \multicolumn{2}{|l|}{$\begin{array}{l}\text { Table 1: Test conditions in IHF arc } \\
\text { jet for the demonstration test }\end{array}$} \\
\hline Main air mass flow $(\mathrm{g} / \mathrm{s})$ & 110 \\
\hline Add air mass flow $(\mathrm{g} / \mathrm{s})$ & 160 \\
\hline Arc current $(\mathrm{A})$ & 2200 \\
\hline 4" hemi heat flux $\left(\mathrm{W} / \mathrm{cm}^{2}\right)$ & 184 \\
\hline 4.5" cyl heat flux $\left(\mathrm{W} / \mathrm{cm}^{2}\right)$ & 149 \\
\hline Stag pressure $(\mathrm{kPa})$ & 7.8 \\
\hline
\end{tabular}

are then transformed into time-dependent boundary conditions for analysis of the MISP response.

Several instrumented PICA models were built and tested to demonstrate the concept. The tests were run in the NASA Ames Interaction Heating Facility (IHF) arc jet. The target stagnation point heat flux and pressure on the calorimeter model established the constant arc jet test condition. The run conditions are listed in Table 1. The rotating model angle vs. time schedule was programmed for the MISP location to approximate the transient heating profile experienced by MSL. Figure 3 illustrates how computational simulations of the heat flux over the cylinder were used to map heat flux vs. time at a point on the MSL vehicle to the rotation angle vs. time of the test article mechanism.

A series of photos from one of the demonstration runs appears in Fig. 4. The MISP plug begins the test run at $90^{\circ}$ to the flow. Immediately after insertion of the test model, the programmed mechanical rotation of the test article is initiated. The succession of images shows the MISP plug rotating towards the stagnation point then back to the initial position as it experiences the transient heat pulse. The total exposure time was approximately 60 seconds.

In a conventional, constant-condition arc jet test, the surface temperature of the TPS material will rise monotonically until the temperature reaches an equilibrium value or the model is removed. Once the model is removed from the stream, the surface temperature falls rapidly as the model cools. For the rotating arc jet test model, the transient applied heat flux pulse generates a different surface temperature response at the target location. The time history of temperatures from the MISP thermocouples is plotted in Fig. 5. The MISP plug has five thermocouples at depths of $0.1 ", 0.2 ", 0.45 ", 0.7$ ", and 1.0 " below the surface. The duration of the model exposure is denoted in the plot. The temperature measured by the first thermocouple nearest the surface peaks then begins to fall during model exposure, which correlates with the applied heat flux pulse at the MISP location. 


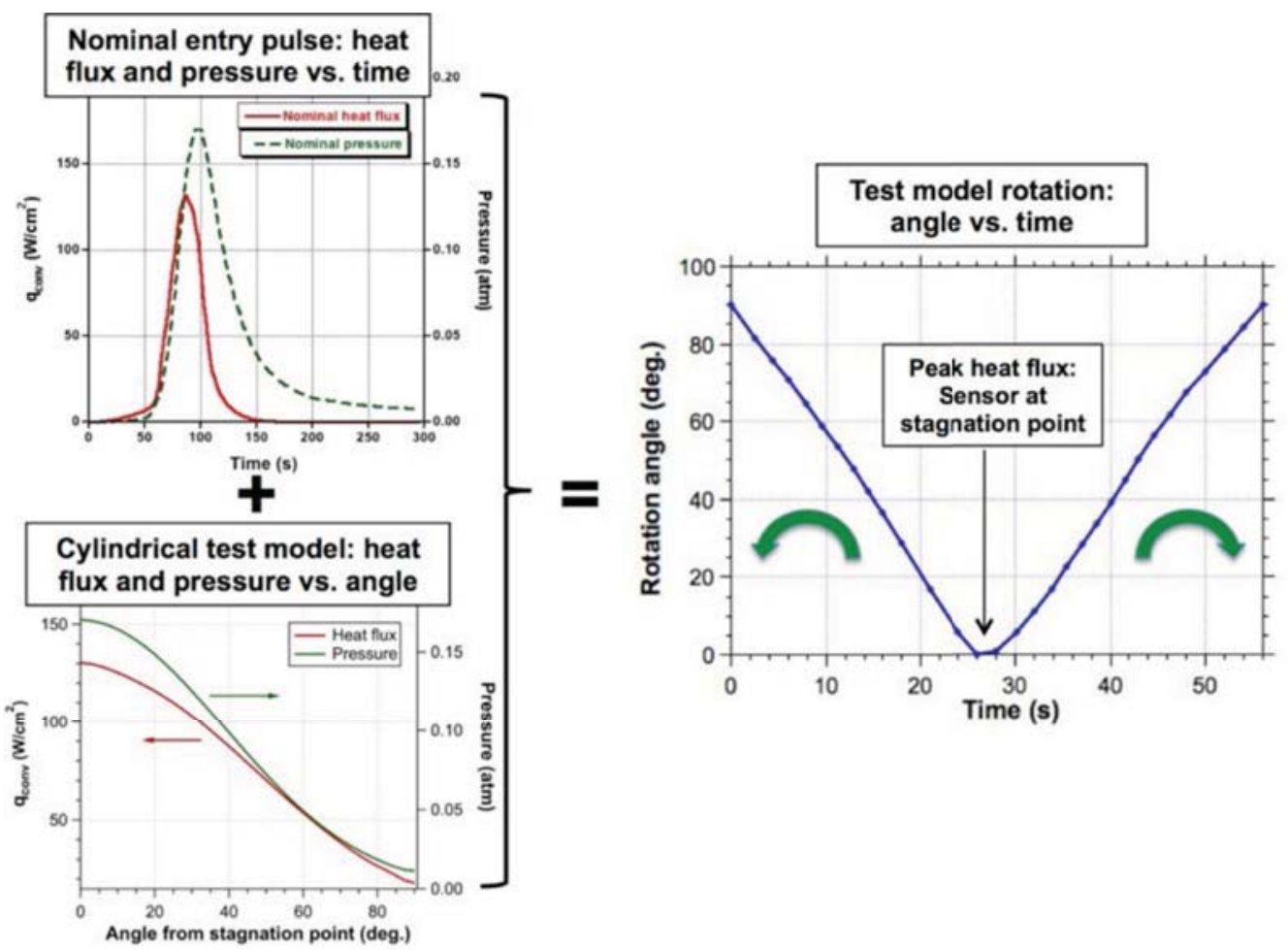

Figure 3: Time-varying arc jet testing methodology: Targeted heat flux pulse and spatial heat flux distribution on cylindrical test model are used to determine model rotation angle vs. time to replicate time-dependent heat flux at instrumented location on test article.

The characteristics of the transient applied heat flux are likewise conveyed through the material's subsurface response in an analogous manner to flight.

The time-varying arc jet test methodology described above can be applied to all stages of mission engineering - materials research and development, qualification and certification testing, flight instrumentation development. For example, the test engineer can formulate tests for evaluation of materials under a variety of conditions, such as flight-like skip and aerocapture/entry trajectories or period modulation for response analysis.

The paper will describe mechanical and thermal engineering design development of the model rotation mechanism, pre-test simulation results, and data from the initial demonstration tests.

\section{Acknowledgments}

Support was provided by NASA's Hypersonics Project/Fundamental Aeronautics Program and MSL/MEDLI Project. The authors thank M. Wright of NASA Ames, M. Munk of NASA Langley, and the engineers and technical staff of the Ames arc jet facility and TPS model development laboratory.

\section{References}

1. Bose, D., Santos, J.A., Rodriguez, E., White, T., Olson, M., and Mahzari, M., "Mars Science Laboratory Heat Shield Instrumentation and Arc Jet Characterization," AIAA Paper 2013-2778, June 2013. 

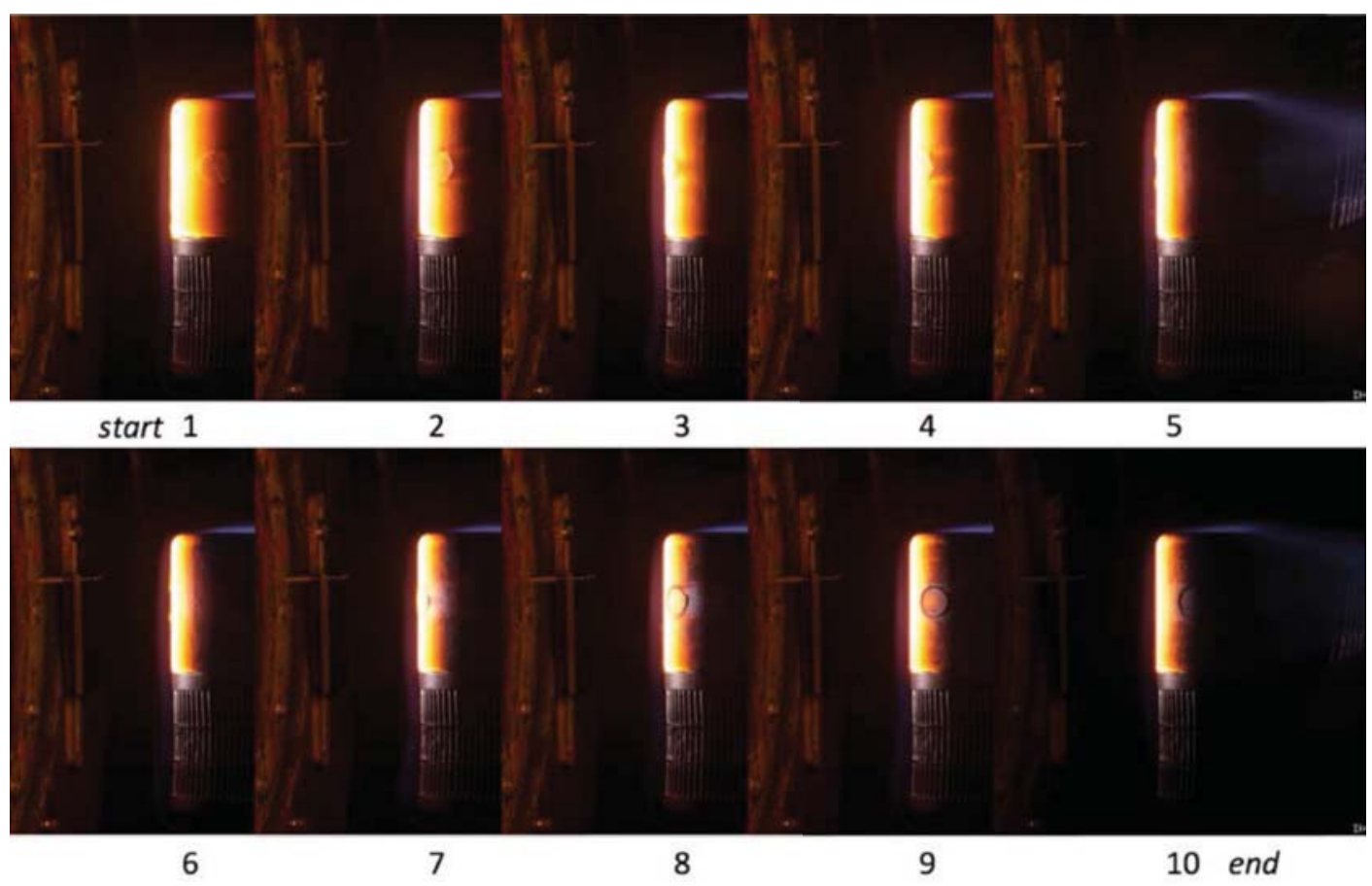

Figure 4: Time sequence images of a rotating arc jet model under test. Flow is from left to right. The MISP embedded in the TPS model faces the camera at the start and end of the test. The model rotates the MISP to the stagnation point then back. The time interval between images is approximately 7 seconds.

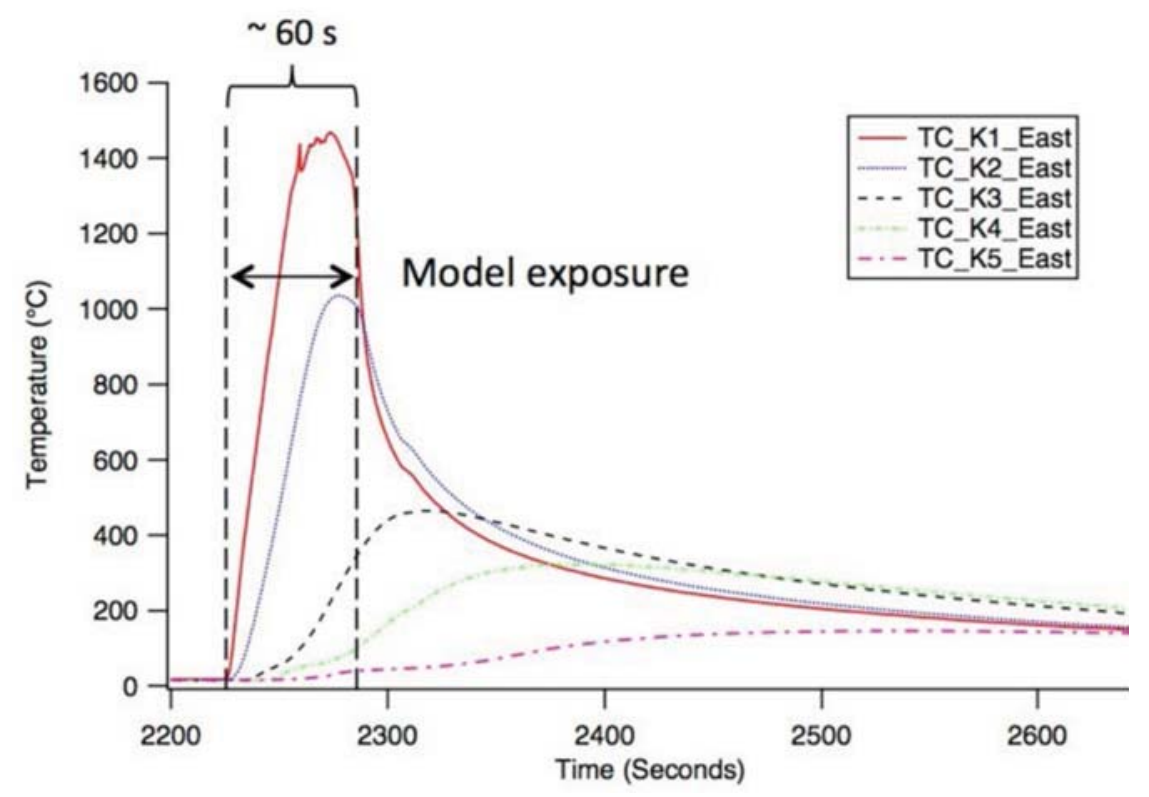

Figure 5: MISP thermocouple time histories during and after rotating arc jet test model exposure. The temperature from the near-surface thermocouple (TC_K1_EAST) reaches a peak value and begins to drop before the model is removed as the applied heat flux also peaks and decreases due to model rotation. 